\title{
Optimized design of engine intake manifold based on 3D scanner of reverse engineering
}

\author{
Shuqing Guo ${ }^{1 *}$, Shuo Huang ${ }^{1}$ and Mingshan $\mathrm{Chi}^{2}$
}

\begin{abstract}
It is difficult to design the engine intake manifold in normal method because of the complex structure, and the real situation of fluid movement in the manifold cannot be obtained through the steady flow test, so the optimized design is proposed about the engine intake manifold based on reverse engineering. A3D model of the intake manifold is build according to the CATIA software; fluid mathematical is set up by computational fluid dynamics method. The 3D geometry model of the intake manifold is grid with the Gambit software and the divided grid data is imported into the Fluent. These are performed about the simulation of the fluid pressure field, velocity field, flow field, and local flow field in the manifold. Key parameters design of the intake manifold relies on the results. It proves that the reverse technology is reasonable for the intake manifold design through the theory and simulation analysis; it can ensure sufficient air intake and reduce the intake resistance and energy loss of the air, and can improve the air intake uniformity.
\end{abstract}

Keywords: Reverse engineering, The engine intake manifold, Optimized design, Computational fluid dynamics, Simulation analysis

\section{Introduction}

With the improvement of people's living standards, the car has become an indispensable travel tool. However, the vehicle structure is supposed to be redesigned continuously to meet energy saving and environmental protection due to the energy shortage and serious air pollution. But, the advanced core technology cannot be grasped in some of the Chinese enterprises. So experts are constantly trying and innovating, and a lot of experiment shows it is possible for reverse engineering to be introduced and gradually put into use in the car design to improve vehicle power, economy, and emission performance.

Due to the complex structure of the intake manifold, it can be modified and optimized according to the experience, and the performance of the intake manifold is only evaluated through the steady flow test in the automobile design. Therefore, it is not helpful to improve the intake manifold design because the test method is limited, uncertain, high cost, and long period, and the flow

\footnotetext{
* Correspondence: guoshuqing0329@126.com

'Faculty of Traffic and Building Engineering, Beihua University, Jilin City, China

Full list of author information is available at the end of the article
}

condition can be neither truly reflected nor the internal flow field can be analyzed in detail in the manifold. So the reverse engineering will be used to improve the intake manifold structure.

The basis of design is to obtain the data. The purpose of design is to deal with the data and set up the improved model.

\section{Basic theory and method}

A mathematical model must be created to reflect its motion in order to analyze the air flow in the intake manifold. A grid model will be established for simulation in the analysis.

\subsection{Reverse engineering}

Reverse engineering (also called reverse technology) is a reproduction process of product design technology, that is, the reverse analysis and research of a target product, so the design parameters will be obtained by studying the product's processing, organizational structure, functional characteristics and technical specifications, etc., then a production is produced that is similar to the original function, but not the same one. In general, to design a product in reverse engineering is the process which the 
design data is introduced backwardly (including various types of design drawings or data models) based on the existing products $[1,2]$.

\subsection{Simulation theory}

The simulation technology is mature for the air movement in the manifold of an engine with the rapid development of the computer technology. The $k-\varepsilon$ models and unstructured grids have been introduced and used, as well as the availability of computational fluid dynamics (CFD) software, such as CFX, FLUENT, STAR-CD, etc., which have greatly optimized the intake manifold design process and accuracy. CFD has gradually become the most effective tool for analyzing the air movement of the engine intake system.

\subsubsection{Computational fluid dynamics}

CFD is an analysis to the fluid flow, thermal conduction, and other related physical phenomena through using the computer numerical calculations and graphical displays [3]. The basic idea of CFD can be summarized as follows: initial physical space or continuous physical field in space domain, for example, the refinement values are used for the variables of each discrete points, which are used to represent continuous fields in the pressure and velocity field. The algebraic equations are created according to the relationship between these discrete points and variables, and the specific rules and methods, and then the corresponding approximate values are obtained by solving the algebraic equations. The basic equation of CFD is build based on the simulation calculation. What the flow of fluids is known by the basic equations that contain conservation of momentum, conservation of energy, conservation of mass, etc. No matter how complex the flow field is, the distribution of basic physical quantities (such as concentration, temperature, pressure, velocity, etc.) at all internal points can also be obtained by using numerical simulation. That can also show the cavitation and distribution characteristics, and the flow area, etc. changing over time and some basic physical quantity. In addition, the product structure can also be designed and optimized in conjunction with CAD.

\subsubsection{CFD calculation procedure}

The numerical simulation of fluid flow using CFD method [4] is shown in Fig. 1.

First of all, the nature must be reflected on a certain physical or engineering problem by establishing mathematical model, that is, the relationship is obtained among the variables based on the research questions.

The first step is the relationship can be accurately reflected among variables, and the conditions needed to solve the equations be determined by establishing differential equations (groups) during the numerical simulation calculations.
Second, the pursuit of fast, high-precision algorithm is needed, that is, the appropriate analysis methods are selected to discretize the numerical value of the corresponding control equations (clusters); the methods are known as finite element method, finite difference method, etc.

Again, calculation procedures and operations is to be done. It includes grid, setting boundary conditions or initial conditions, and inputting control parameters, etc.

Finally, the calculation results are displayed. The results are usually expressed using graphs or tables, which is of great significance for analyzing and judging the accuracy of calculation results.

Usually, the practical problems can be solved through CFD software. However, if the problems are more complicated and CFD software is difficult to meet the calculation requirements, users need to write their own calculation programs. The general analysis process is the same regardless of the method.

\subsubsection{FLUENT software}

A calculation program is basically written by user in order to complete the simulation calculation before the CFD software is applied. The degree of correlation is limited among the program written by user due to the diversity of computer hardware and the complexity of computational fluid dynamics, resulting in a waste of resources. The CFD is easily edited into generic software because of its systematicity and regularity. FLUENT is very versatile to perform numerical conversions [5]. The various problems can be solved for each field if it can be combined with other CFD software. And it can also link with various drawing software.

Automate drawing cannot be done by the FLUENT software because the model is relatively complex. You can import the 2D and 3D models drawn by CATIA into the preprocessor GAMBIT for grid. The conditions of the initial and boundary are set to solve the model, thus a lot of time can be saved. It is more convenient to draw a simple model directly from GAMBIT. The grid model with well-defined boundary conditions is imported into the solver. The appropriate solution method is selected by FLUENT based on the specific flow problem, and the solving equation is determined accordingly, which make the calculation process stable and the result more accurate. Finally the basic information is displayed for the flow field through the post-processing module, such as the speed vector, pressure cloud map, kinetic energy cloud map, and the other information required by the user, such as contour maps (including streamline maps, isobaric maps, etc.). The XY-PLOT function can be also used to represent the values of various physical 


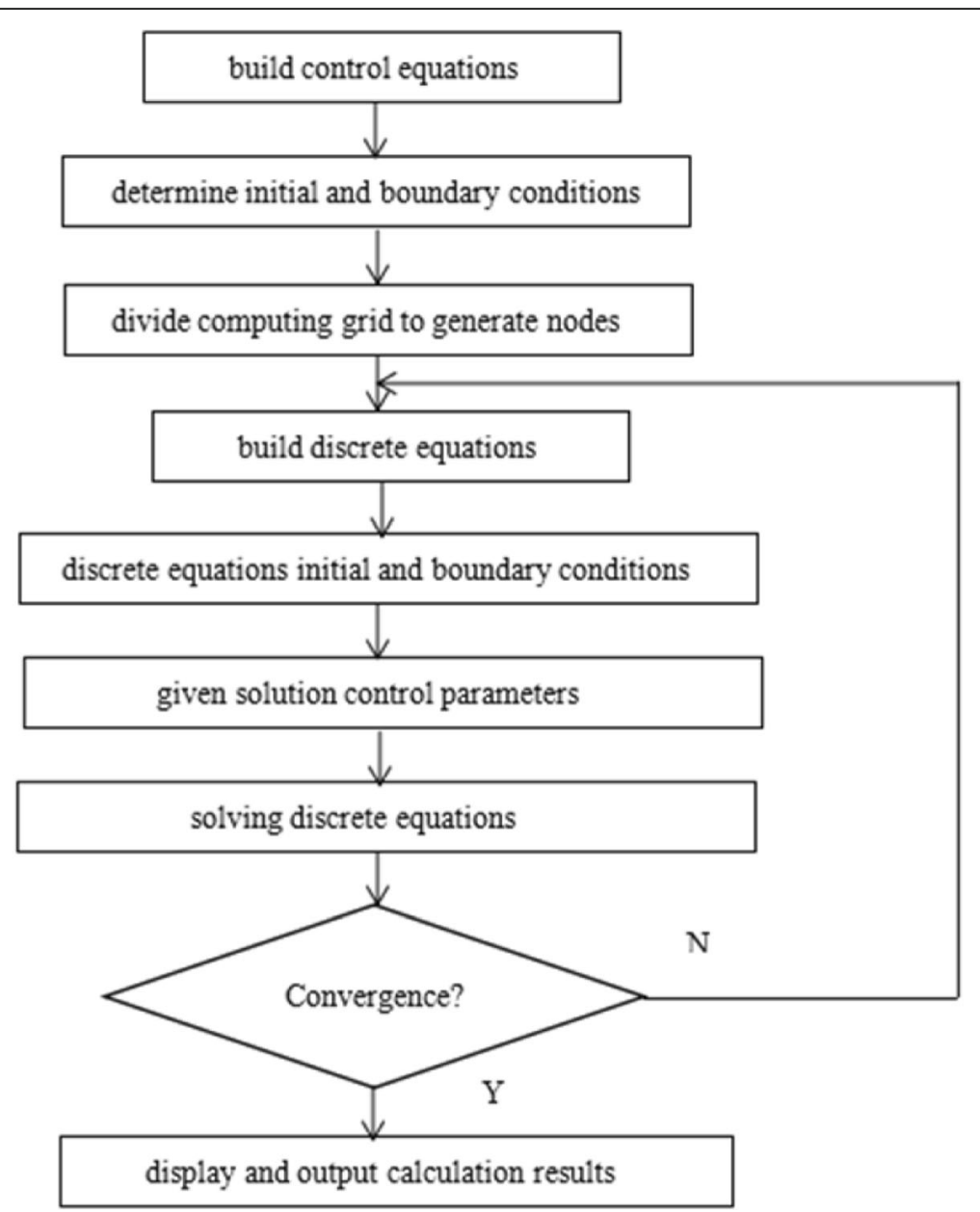

Fig. 1 CFD procedure

quantities (such as pressure, velocity, temperature, etc.) along a certain line or point on a plane in the computational space domain.

\subsection{Air motion equation}

The Navier-Stokes equations are the basic equations that all fluid motions must follow [6]. Compressible viscosity is a common characteristic of real fluids. In this paper, the air is taken as the research object, and the fluid in the manifold is regarded as a three-dimensional compressible viscous fluid. The control equations must conform to the general hydrodynamic equations and should satisfy: mass conservation equation, momentum conservation equation, and energy conservation equation.

\subsubsection{Mass conservation equation}

Mass conservation law: The increase in mass in a fluid-time micro-unit per time is equal to the net mass flowing into the micro-cell at the same time interval. According to this law, the equation of mass conservation can be drawn [4].

$$
\frac{\partial \rho}{\partial \mathrm{t}}+\frac{\partial(\rho \mathrm{u})}{\partial \mathrm{x}}+\frac{\partial(\rho \mathrm{v})}{\partial \mathrm{y}}+\frac{\partial(\rho \mathrm{w})}{\partial \mathrm{z}}=0
$$

Equation (1) represents a transient compressible fluid. If the fluid cannot be compressed, the density is constant, and Eq. (1) can be written as:

$$
\frac{\partial u}{\partial x}+\frac{\partial v}{\partial y}+\frac{\partial w}{\partial z}=0
$$

If the flow is stable, the density does not change with time. Equation (1) can be written as:

$$
\frac{\partial(\rho \mathrm{u})}{\partial \mathrm{x}}+\frac{\partial(\rho \mathrm{v})}{\partial \mathrm{y}}+\frac{\partial(\rho \mathrm{w})}{\partial \mathrm{z}}=0
$$

\subsubsection{Conservation of momentum equation}

The law of conservation of momentum can be described as the rate to time of change of the momentum of the fluid in the micro-body is equal to the sum of the external forces acting on the element. According to this law, 
the momentum conservation equations can be found in the three directions $x, y$, and $z$ [4]:

$$
\begin{aligned}
& \frac{\partial(\rho u)}{\partial t}+\frac{\partial(\rho u u)}{\partial x}+\frac{\partial(\rho u v)}{\partial y}+\frac{\partial(\rho u w)}{\partial z} \\
& =\frac{\partial}{\partial x}\left(u \frac{\partial u}{\partial x}\right)+\frac{\partial}{\partial y}\left(u \frac{\partial u}{\partial y}\right)+\frac{\partial}{\partial z}\left(u \frac{\partial u}{\partial z}\right)-\frac{\partial p}{\partial x}+S_{u} \\
& \frac{\partial(\rho \mathrm{v})}{\partial t}+\frac{\partial(\rho v u)}{\partial x}+\frac{\partial(\rho v v)}{\partial y}+\frac{\partial(\rho v w)}{\partial z} \\
& =\frac{\partial}{\partial x}\left(u \frac{\partial v}{\partial x}\right)+\frac{\partial}{\partial y}\left(u \frac{\partial v}{\partial y}\right)+\frac{\partial}{\partial z}\left(u \frac{\partial v}{\partial z}\right)-\frac{\partial p}{\partial y}+S_{v} \\
& \frac{\partial(\rho \mathrm{w})}{\partial t}+\frac{\partial(\rho w u)}{\partial x}+\frac{\partial(\rho w v)}{\partial y}+\frac{\partial(\rho w w)}{\partial z} \\
& =\frac{\partial}{\partial x}\left(u \frac{\partial w}{\partial x}\right)+\frac{\partial}{\partial y}\left(u \frac{\partial w}{\partial y}\right)+\frac{\partial}{\partial z}\left(u \frac{\partial w}{\partial z}\right)-\frac{\partial p}{\partial z}+S_{w}
\end{aligned}
$$

where $\mu$ is the dynamic viscosity and $S_{\mathrm{u}}, S_{\mathrm{v}} S_{\mathrm{w}}$ are the generalized source term of the conservation of momentum equation; $S_{\mathrm{u}}=F_{x}+S_{x}, S_{\mathrm{v}}=F_{\mathrm{y}}+S_{y}, S_{\mathrm{w}}=F_{z}+S_{z}$ and the expression of $\mathrm{s}_{\mathrm{x}}, \mathrm{s}_{\mathrm{y}}, \mathrm{s}_{\mathrm{z}}$ is as follows:

$$
\begin{aligned}
s_{x}= & \frac{\partial}{\partial x}\left(\mu \frac{\partial \mu}{\partial x}\right)+\frac{\partial}{\partial y}\left(\mu \frac{\partial v}{\partial x}\right)+\frac{\partial}{\partial z}\left(\mu \frac{\partial w}{\partial x}\right) \\
& +\frac{\partial}{\partial x}(\lambda d i v u) \\
s_{\mathrm{y}}= & \frac{\partial}{\partial x}\left(\mu \frac{\partial \mu}{\partial y}\right)+\frac{\partial}{\partial y}\left(\mu \frac{\partial v}{\partial y}\right)+\frac{\partial}{\partial z}\left(\mu \frac{\partial w}{\partial y}\right) \\
& +\frac{\partial}{\partial y}(\lambda d i v u) \\
s_{\mathrm{z}}= & \frac{\partial}{\partial x}\left(\mu \frac{\partial \mu}{\partial \mathrm{z}}\right)+\frac{\partial}{\partial y}\left(\mu \frac{\partial \nu}{\partial z}\right)+\frac{\partial}{\partial z}\left(\mu \frac{\partial w}{\partial z}\right) \\
& +\frac{\partial}{\partial z}(\lambda d i v u)
\end{aligned}
$$

The above formulas are valid for all fluids (including non-Newtonian fluids).

\subsubsection{Energy conservation equation}

The law of conservation of energy is the basic law that must be satisfied by the flow system of heat exchange.

The law can be expressed as the rate of increase of energy in the microelement is equal to the net heat flux entering the microelement plus the work done by the physical force and surface force on the microelement. The internal energy $i$, kinetic energy $K=\left(\mathrm{u}^{2}+\mathrm{v}^{2}+\mathrm{w}^{2}\right) / 2$, and potential energy pare summed to obtain the fluid energy $E$. Therefore, the energy conservation equation is listed for the total energy $E$ with the temperature $T$ as a variable [7]:

$$
\frac{\partial(\rho T)}{\partial \tau}+\operatorname{div}(\rho u T)=\operatorname{div}\left(\frac{k}{c_{\mathrm{p}}} \operatorname{grad} T\right)+S_{T}
$$

In the formula:

$\mathrm{c}_{\mathrm{p}}$ - specific heat capacity

K-heat transfer coefficient

T-temperature

i-internal energy

$i=\mathrm{c}_{\mathrm{p}} \mathrm{T}$

$S_{T}$-internal heat source and part of mechanical energy converted into heat energy due to stickiness.

\subsection{Turbulence model}

In nature, turbulent flow is a relatively common phenomenon of fluid movement. In engineering, turbulent flow is also a common phenomenon in fluids, and turbulence characteristics are very important. The numerical simulation method is mainly introduced to solve the turbulence phenomena existing in the engine intake manifold [8]; it focuses mainly on the perspective of engineering applications in this paper.

\subsubsection{Basic characteristics of turbulent flow}

The kinetic experiment proves the flow is smooth when the Reynolds number is at a certain critical value, and the fluid layer is ordered and does not have influence between each other. Therefore, it is also called laminar flow. When the Reynolds number is higher than the critical value, the flow is in an un-ordered state, because a series of changes has been occurred to change the orderly flow. At this time, although the boundary conditions can be maintained unchanged, the flow characteristics is altered due to the unstable flow state, so it is known as a turbulent flow. The variation of the velocity changes with time in the flow field under turbulent flow condition is shown in Fig. 2. It can be seen from the figure that the speed value is not stable and the pulsation is very strong. The engineering design will be affected by the pulsation in the turbulent flow. The pulsating pressure will increase the instantaneous load of the wind speed that the object can withstand. The fluctuation will be created because of the largest negative peak caused by pressure fluctuations in pulsation pressure for the engine intake manifold.

\subsubsection{Basic equations of turbulent flow}

Although turbulent motion is very complex, it is generally believed that the unsteady continuity equation and Navier-Stokes are still valid for relatively instantaneous turbulent motion. In normal, the time-average method can be used to obtain the effect of pulsation, which is considered as a superposition of the time-averaged flow and the time-averaged flow of the instantaneous pulsating flow. Therefore, the turbulent time-averaged control equation can be written as: 


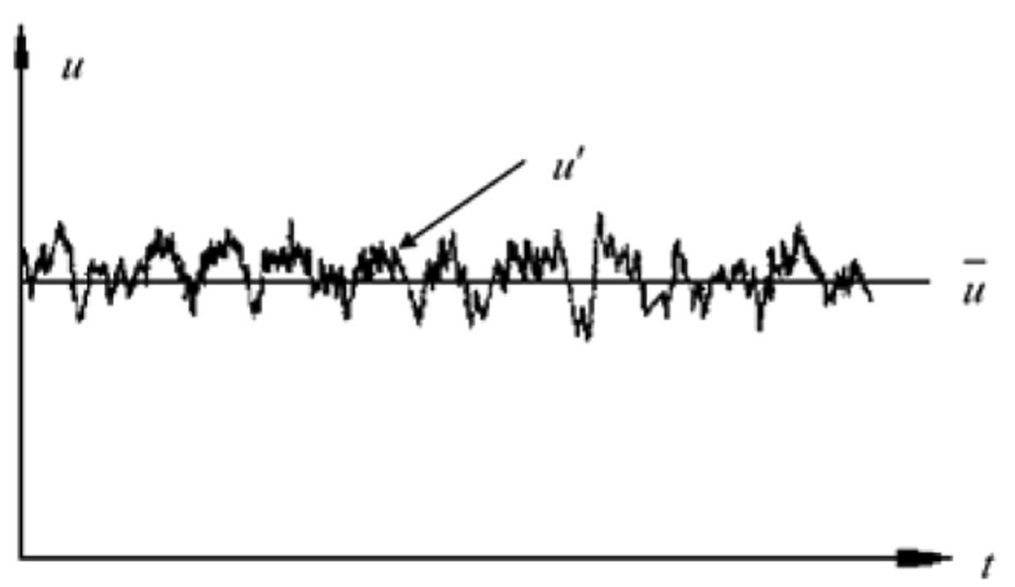

Fig. 2 Measured speed of turbulence at a certain point

$$
\frac{\partial \rho}{\partial t}+\frac{\partial}{\partial x_{i}}\left(\rho u_{i}\right)=0
$$

Navier-Stokes equation:

$$
\begin{gathered}
\frac{\partial}{\partial \mathrm{t}}\left(\rho \mathrm{u}_{\mathrm{i}}\right)+\frac{\partial}{\partial x_{j}}\left(\rho u_{i} u_{j}\right)=-\frac{\partial p}{\partial x_{i}} \\
+\frac{\partial}{\partial x_{j}}\left[\mu\left(\frac{\partial u_{i}}{\partial x_{j}}+\frac{\partial u_{j}}{\partial x_{i}}-\frac{2}{3} \delta_{i j} \frac{\partial u_{i}}{\partial x_{i}}\right)-\rho \overline{u_{i}^{\prime} u_{j}^{\prime}}\right]
\end{gathered}
$$

Where $-\rho \overline{u_{i}^{\prime} u_{j}^{\prime}}$ is unknown Reynolds stress component; $i, j=1,2,3$.

It can be seen from Eq. (12), new ripple-related items $-\rho \overline{u_{i}^{\prime} u_{j}^{\prime}}$ appears in the flow equation in a temporally even form, defining this item as a Reynolds stress term; its appearance prevents the system of equations from being closed. That is to say $-\rho \overline{u_{i}^{\prime} u_{j}^{\prime}}$, the different closed equations obtained by different simulation methods, it will form different turbulence models.

\subsubsection{Turbulence model}

Turbulence models can be divided into single equation model, double equation model, algebraic Reynolds stress model, differential Reynolds stress model, low Reynolds number turbulence model, etc.; $k-\varepsilon$ is one of the control equations of air motion. The standard $\mathrm{k}-\varepsilon$ model is as follows: Turbulent kinetic energy equation (k)

$$
\begin{aligned}
& \frac{\partial(\rho \varepsilon)}{\partial \mathrm{t}}+\frac{\partial}{\partial x_{j}}\left(\rho u_{j} k-\frac{\mu_{e f f}}{\sigma_{k}} \frac{\partial k}{\partial x_{j}}\right) \\
& =u_{i}\left(P+P_{B}\right)-\rho \varepsilon-\frac{2}{3}\left(\mu_{i} \frac{\partial \mu_{i}}{x_{i}}+\rho k\right) \frac{\partial u_{i}}{\partial x_{i}}
\end{aligned}
$$

Turbulence dissipation rate model $(\varepsilon)$ :

$$
\begin{gathered}
\frac{\partial(\rho \varepsilon)}{\partial \mathrm{t}}+\frac{\partial}{\partial x_{\mathrm{i}}}\left(\rho u_{j}-\frac{\mu_{\text {eff }}}{\sigma_{k}} \frac{\partial \varepsilon}{\partial x_{i}}\right) \\
=\frac{\varepsilon}{k}\left[\left(C_{\varepsilon 1} P+C_{\varepsilon 3} P_{B}\right)-\frac{2}{3}\left(\mu_{t} \frac{\partial \mu_{i}}{\partial x_{i}}+\rho k\right) \frac{\partial u_{i}}{\partial x_{i}}\right] \\
-C_{\varepsilon 2} \rho \frac{\varepsilon^{2}}{k}+C_{\varepsilon 4} \rho \varepsilon \frac{\partial u_{i}}{\partial x_{i}} \\
\mu_{\text {eff }}=\mu_{t}+\mu
\end{gathered}
$$

where $\mu$ is dynamic viscosity coefficient and $\mu_{t}$ is turbulent viscosity coefficient; $\mu_{\text {eff }}$ is effective viscosity coefficient; $\mu_{\mathrm{i}}$ is the component of the airflow velocity on the three coordinates; $P$ is average stress term; $P_{B}$ is physical items; $\sigma_{k}$ is $k$ turbulence Prandtl number; and $\sigma_{\varepsilon}$ is $\varepsilon$ turbulence Prandtl number.

\subsection{SIMPLE algorithm}

Since1972, the SIMPLE algorithm has been widely used in the field of computational fluid dynamics and related fields [4]. It has quickly become the primary algorithm for calculating the flow conditions of incompressible flow fields. It has been successfully applied to the computation of compressible flow fields and has become a numerical simulation method that can calculate fluid flow at any flow rate.

SIMPLE algorithm solution steps are as follows:

(1) Let us assume a speed, and write this as $u_{0}, v_{0}, w_{0}$, and calculate the coefficients and constants in the discrete equation of momentum;

(2) Assuming a pressure field $p_{0}$;

(3) In order to get the momentum equation $u_{1}, v_{1}, w_{1}$;

(4) Correct the pressure and get $p_{1}$;

(5) According to $p_{1}$ to improve speed value; 
(6) After the velocity field is modified, the variable $\Phi$ is found through the source properties coupling to the velocity field. If the flow field is not affected by the variable $\Phi$, the velocity field should be solved after convergence.

(7) A new coefficient of the momentum discrete equation will be calculated after the velocity field is improved, then the calculated value of the pressure field can be used as the initial value of the next layer of calculation. Repeat the above steps until you get a converged solution.

\section{Simulation method of air flow in the engine intake manifold}

The solid model has been obtained through the reverse method before the value is analyzed for the engine intake manifold. At the same time, the simplified Gambit model is used to divide the solid model of the grid so that the analysis can be fully satisfied for the CFD application software. Boundary condition is determined to prepare simulation.

\subsection{GAMBIT simulation method}

The core of solution fluid mechanics is the discrete point of the control equation (such as the finite difference method) or the discrete area (such as the finite element method), and then it is converted into an algebraic equation group. If the equation is nonlinear, the linearization is needed, and then the algebraic iteration method is used to solve.

A three-dimensional solid grid must be generated to solve the problem which the object flows in the three-dimensional space [9]. There are five ways to generate volumetric grid in GAMEBFT: mapping grid method, sub-mapping grid method, Cooper method, T grid method, and hybrid grid method.

\subsection{Method to divide the grid}

Through the above analysis, Tri in elements is selected as the surface grid, and Pave in type; TET/Hybrid in elements are selected as the body grid, TGrid in type, and Spring is 3.5 .

It is relatively difficult to obtain the regular because of complex model structure. Therefore, the amount of computation of the CPU is reduced by using a grid which the variation is relatively small in the density [10].

When the boundaries is initially defined for the grid, all manifold surfaces are selected as walls, and use one of the air inlet surfaces as the pressure inlet, and make the rest pressure outlets. Divided grid is shown in Fig. 3.

\subsection{Setting boundary conditions}

A key step in numerical analysis is numerical simulation. Reasonable boundary conditions can not only

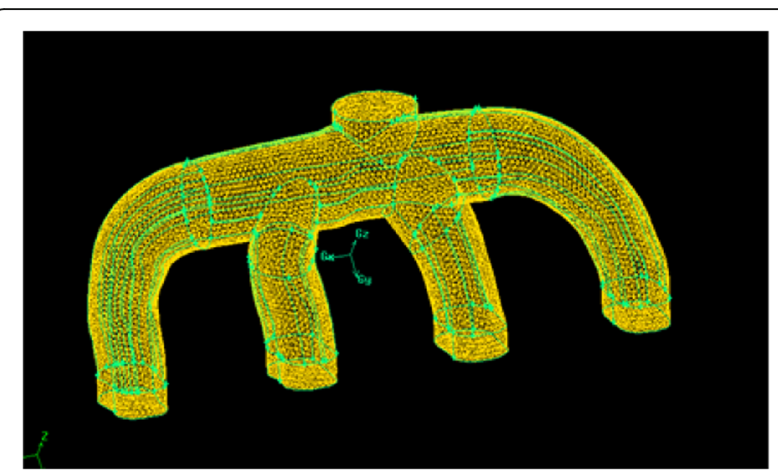

Fig. 3 Intake manifold GAMBIT grid

accelerate the convergence of calculations, but also cause no divergence and overflow of calculation. The so-called boundary condition is the rule that the variable or its first derivative is changed over time on the boundary of the solution domain. Including flow import boundary, flow exit boundary, wall boundary, etc. In the actual operation of the engine, the air flow in the general manifold can be defined as three-dimensional, unsteady, and compressible gas such as heat transfer and friction, but in the calculation simulation, the air flow needs to be considered steady state, and a compressible ideal gas. Therefore, boundary conditions are set for wall, inlet, and outlet.

\subsubsection{Fixed wall boundary}

In normal, material roughness is small for the engine intake manifold; the wall surface can be smooth when it is analyzed to the flow field of the intake manifold, and no slip boundary. The heat exchange is very fast between the gas and the pipe wall. Therefore, the thermodynamic performance of the wall is simplified to a thermal insulation wall. So, the fixed wall boundary conditions are adiabatic and non-slip, fixed temperature is $293 \mathrm{~K}$, and the boundary layer is treated with a turbulent wall function.

\subsubsection{Inlet boundary}

The inlet boundary data is set: intake flow rate $15 \mathrm{~m} /$ $\mathrm{s}$, inlet diameter $0.0504 \mathrm{~m}$. By the formula $\operatorname{Re}=\frac{U \mathrm{UD}_{\mathrm{i}} \rho}{\mu}$, $U$ is import speed, $D_{\mathrm{i}}$ is inlet diameter, $\rho$ is $1.225, \mu$ is $1.7894 * 10^{-5}$, Reynolds number is 51754.778 . By the formula $0.16 * \mathrm{R}_{\mathrm{e}}^{-1 / 8}$, the calculated turbulence intensity is 4 , the hydraulic diameter is $0.0504 \mathrm{~m}$, and the inlet temperature is $293 \mathrm{~K}$.

\subsubsection{Outlet boundary}

The outlet boundary data is set: the outlet diameter is $0.035 \mathrm{~m}$ and the return turbulence intensity is 5 . 


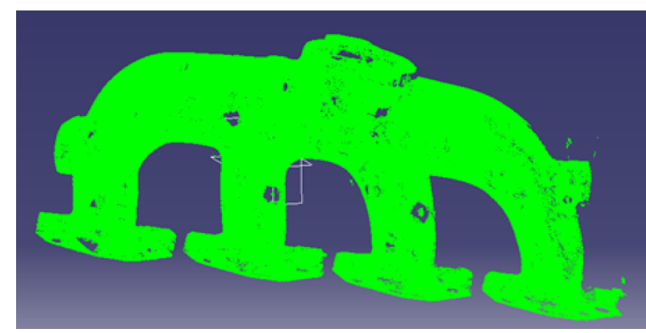

figure before processing

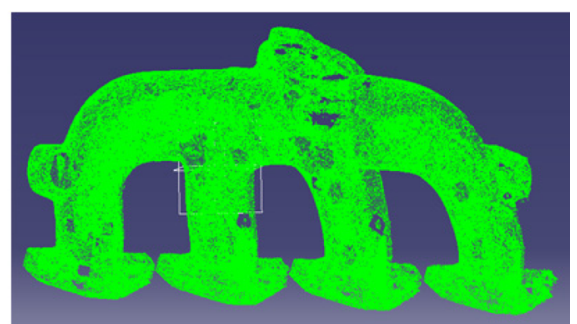

figure after processing

Fig. 4 Intake manifold point cloud

\section{Results and discussion of intake manifold}

A non-contact 3D scanner is used to obtain point cloud data, which is deleted and filtered to get the intake manifold point cloud, and by using CATIA point cloud import function to get the intake manifold cloud picture $[11,12]$.

\subsection{Intake manifold surface reconstruction}

The point cloud data obtained by using the 3D method is very large, and it has certain residual defects and noise points, which will have a certain impact on the reconstruction process of the later curves and surfaces $[13,14]$. Therefore, the point cloud data needs to be processed. Before and after processing, the picture is shown in Fig. 4.

The next step is to rebuild the intake manifold surface from the point cloud, which is the most central issue of the entire reverse engineering. First, the measurement points are fitted and converted into surfaces, finally the 3D models are reconstructed by the operation of transition, splicing, and cutting of the surface patches. Inverse design of intake manifold is used CATIA V5 [15]. Surface reconstruction process as shown 5 .

\subsection{Boundary condition settings}

In order to facilitate the air intake comparison of each manifold, the air intake and an air outlet are set for the boundary of the cylinder in the calculation. The other boundary conditions are set as the wall conditions. All calculations use the same boundary conditions. The calculations are performed for the outlet 1 , the outlet 2 , the outlet 3 , and the outlet 4 , respectively. The flow calculation scheme is listed in Table 1.

A grid analysis is performed on the manifold model by using the Gambit software, and import the partitioned grid into FLUENT. When the result converges, it will actively pause the calculation and display the hint on the monitor; if the calculation cannot be stopped in the example, convergence value is set at 0.001 for pressure, momentum, turbulent kinetic energy, and dissipative rate. Therefore, when the all residuals parameters are below $10^{-3}$, the calculation can be considered as the convergence criterion (Fig. 5).
The calculation results is processed by using FLUENT's own post-processing function and the cloud maps are obtained, such as pressure, absolute pressure, turbulent kinetic energy, and turbulent energy dissipation rate maps which are used to analyze the flow field.

The characteristics is analyzed on the velocity and pressure of intake manifold to study the air resistance, and the factors that produces air resistance, and the method of reducing the resistance will be found; the performance of the engine can be improved through increasing the intake.

The local loss in the intake manifold is mainly focused on the flow field. If the air flow is affected by the size, direction, and shape of the flow channel, vortices will be created, the velocity of the air is redistributed, and the fluid particles will be received drastic collision during acceleration and deceleration, resulting in momentum reduction.

The principle of reducing losses is to minimize the generation of vortex or whirlpool areas and to reduce the scope of secondary flow ripples, thereby the momentum exchange caused collision and fluid particle velocity can be reduced during the redistribution process.

Basic measures are to try to adopt a gradual or tapering structure at the pipe diameter, while avoiding the sudden increase and reduction in pipe diameter. Therefore, the following aspects are analyzed according to the analysis results of the intake manifold.

(1) The inlet and outlet are connected with the pressure stabilization room, and the pressure and velocity distribution at the connection;

(2) Distribution of pressure and flow fields in the stabilization tank and manifold;

Table 1 Manifold air flow scheme

\begin{tabular}{lllll}
\hline Scheme & Outlet1 & Outlet2 & Outlet3 & Outlet4 \\
\hline Scheme 1 & Open & Close & Close & Close \\
Scheme 2 & Close & Open & Close & Close \\
Scheme 3 & Close & Close & Open & Close \\
Scheme 4 & Close & Close & Close & Open \\
\hline
\end{tabular}



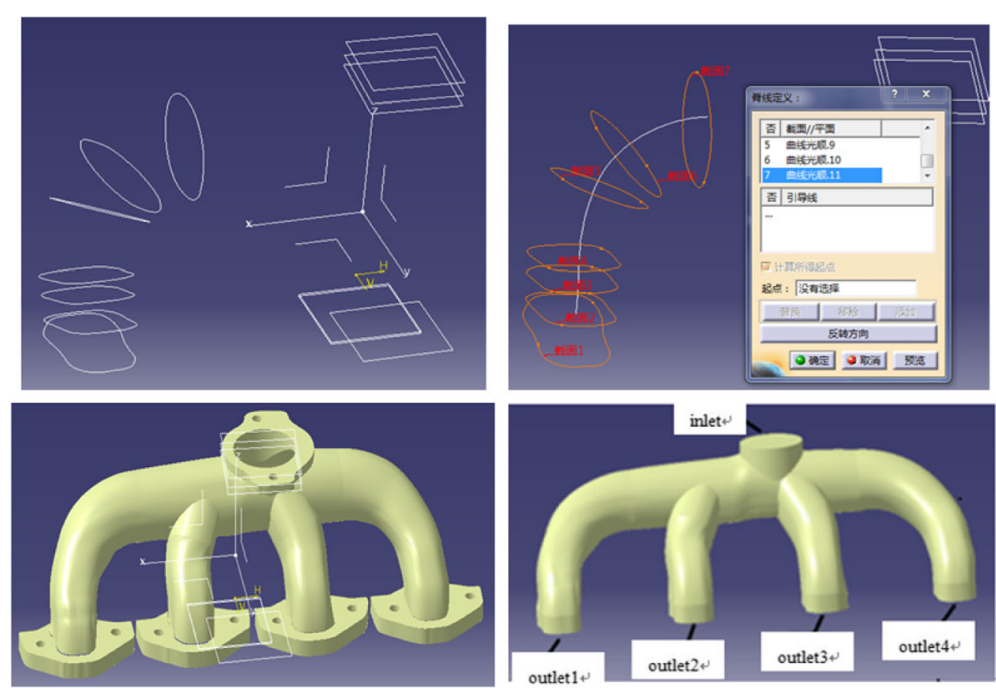

Fig. 5 Surface reconstruction process

(3) Corner pressure and flow field distribution.

Intake manifold non-uniformity formula:

$E_{S}=\left(Q_{\max }-Q_{\text {min }}\right) / Q_{\text {mean }}(16)$.

In the formula:

$Q_{\max }$-maximum intake manifold flow rate $(\mathrm{kg} / \mathrm{s})$;

$Q_{\text {min }}$-minimum intake manifold flow rate $(\mathrm{kg} / \mathrm{s})$;

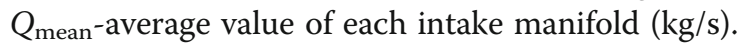

The residual record is observed before analyzing the simulation results, as shown in Fig. 6.

Recorded by the residual error:
The CFD simulation calculation is relatively stable, the convergence speed is fast, and the calculation results are higher quality. This result can be used as the basis for optimizing the manifold structure.

\subsection{Discussion of pressure field in intake manifold}

On the one hand, the reason for the air flow is the pressure difference by analyzing the distribution of the manifold pressure field, and what can be obtained is the direction, size, and distribution of the air flow in the manifold. On the other hand, it is useful to evaluate the

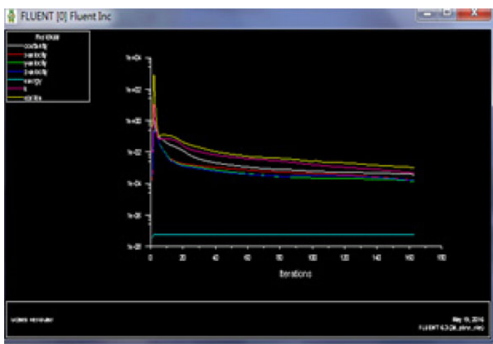

manifold 1 residual plot

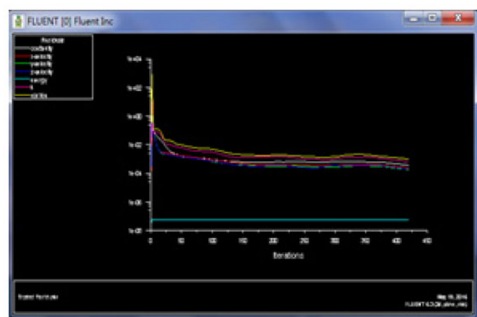

manifold 3 residual plot

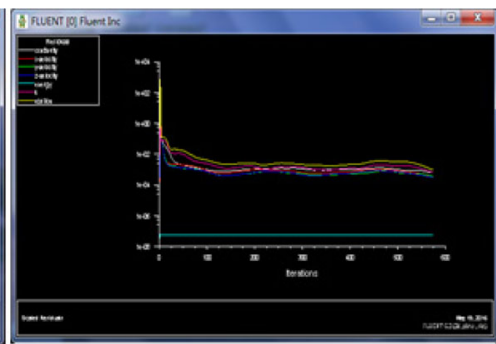

manifold 2 residual plot

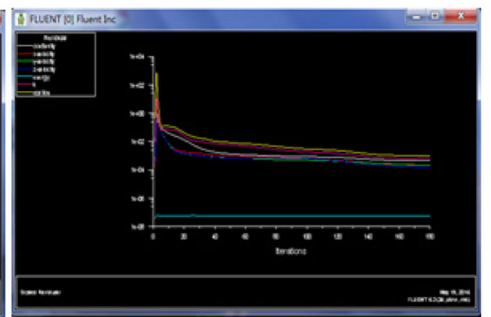

manifold 4 residual plot

Fig. 6 Residual record 
Table 2 Each intake manifold flow

\begin{tabular}{lllll}
\hline Intake manifold number & 1 & 2 & 3 & 4 \\
\hline Flow $Q\left(10^{-2} \mathrm{~kg} / \mathrm{s}\right)$ & 3.65 & 3.34 & 5.17 & 4.13 \\
\hline
\end{tabular}

strength of manifold material whether or not it can withstand the pressure during the intake process.

What can be acquired is shown below:

(1) The variation of the pressure in the manifolds is strikingly coherent;

(2) The pressure of the manifold decreases from the entrance to the exit (except bends), with mathematical calculations; inlet pressure is approximately5.69E $+02 \mathrm{~Pa}$, outlet 1 is $2.74 \mathrm{E}+$ $01 \mathrm{~Pa}$, outlet 2 4.37E + $01 \mathrm{~Pa}$, outlet $31.34 \mathrm{E}+01 \mathrm{~Pa}$, and outlet $41.53 \mathrm{E}+01 \mathrm{~Pa}$.

(3) The faster the air flows, the lower the pressure is in the manifold. Therefore, the air flow decreases, forming a pressure accumulation, and the pressure outside is basically slightly larger than the inside, but the difference is not obvious due to the air flow direction suddenly changes at the four manifold bends.

(4) Air flow will form stress concentrations at the corners, such as the connection from the stable pressure box to the manifold. It is the important part to improve the structure. Smooth transitions can be used during optimization; the method to reduce resistance is the trumpet shape.

\subsection{Discussion of velocity field in intake manifold}

A speed vector is used to analyze the intake manifold speed field; this not only analyzes the dispersion of the speed in the entire intake manifold but also the direction of the air flow can be known according to the arrow direction; in addition, the flow rate is indicated through the length and color of the arrow, and simultaneously the change of the internal flow field is demonstrated in the manifold.

(1) The air flow is basically the same in the various manifolds. The airflow is low at the inlet. However, the flow rate increases due to the smaller diameter, which makes the flow rate at the outlet higher when the air flows through each manifold;

(2) The flow rate values are gradually increasing from the inlet to the outlet;

(3) The flow rate changes more at each corner of the intake manifold. The inlet speed is about $16.7 \mathrm{~m} / \mathrm{s}$, outlet 1 is $23.2 \mathrm{~m} / \mathrm{s}$, outlet 2 is $21.2 \mathrm{~m} / \mathrm{s}$, outlet 3 is $22.9 \mathrm{~m} / \mathrm{s}$, and outlet 4 is $22.3 \mathrm{~m} / \mathrm{s}$;

(4) The outside flow velocity is almost greater than the inside for each intake manifold.
4.5 Discussion of internal and local flow field in manifold What the flow field can be seen on it when a vertical plane is inserted in the middle of the tank, which is used to analyze the air flow inside the tank [16]. What the gas flow is to influence greatly on the air intake and uniformity of the manifold. The part of the air reaches the cylinder across the manifold and the other part moves along the wall of the tank, finally forming a vortex in the tank after the air enters the manifold [17].

(1) The vortex area is not directly above the selected manifold;

(2) The return air flow does not interfere with the intake air and can follow the second airflow into the manifold. This played a very good role of steady pressure and ensures the stability of the air flow.

It is known that the pressure in the field where the airflow cannot reach is high, so that an upward vector is formed in the manifold by analyzing the local flow field. The more gradients are formed and the larger pressure losses are created because of the larger curvatures of the pipe in the manifold 3 and 4 . The result through calculating is listed in Table 2.

Known from the table, it is up to $12.21 \%$ of the inequality in the manifold. The main reason for this phenomenon is the unreasonable design, the size, and the improper transition joints, and the larger curvature of the manifold. Therefore, the smoothness is poor. So improvement design is necessary for the sufficient air and the engine performance.

\section{Method of improved design to the intake manifold}

The collected grid data is calculated and analyzed by the hydrodynamic model and flow field analysis software. The shape and structure are optimized according to the intake manifold based on the results [18].

\subsection{Optimization method of intake manifold structure}

According to the previous analysis, the curvature of the original manifold is small, and the intake resistance is reduced by increasing the curvature of the manifold, which makes the intake air smooth. At the same time, the volume of the stable tank is increased appropriately to reduce the eddy current intensity formed in the manifold.

The GAMBIT software is used to grid the improved intake manifold to generate a calculation domain [19]. The grid model is shown in Fig. 7.

\subsection{Simulation experiment of improved manifold}

The grid model of the optimized manifold is simulated and calculated. The boundary conditions are the same as those 


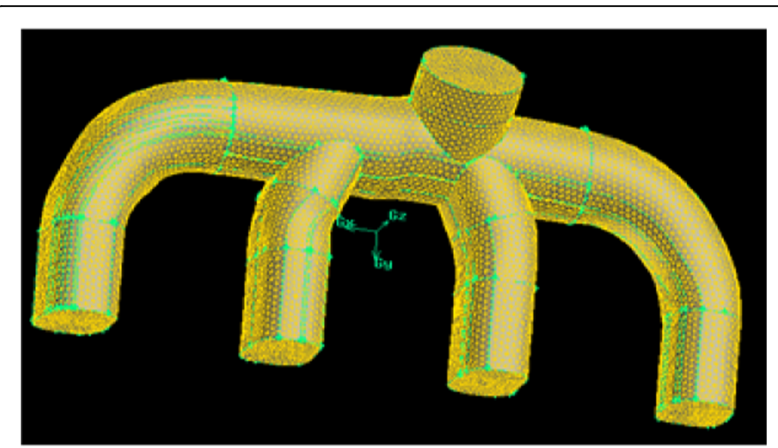

Fig. 7 Improved manifold grid model

of the original manifold, and the calculation steps are unchanged. The calculated residual map is shown in Fig. 8.

The CFD simulation calculation is relatively stable, the convergence speed is faster, and the obtained result is accurate from the monitoring and residual records [20]. The manifold structure can be optimized according to the result. The analysis is to be done about the pressure field of the intake manifold. The manifold pressure field is shown in Fig. 9.

Learn from the above:

(1) Pressure change is basically the same in each manifold. The pressure decreases from the entrance to the exit (except bends);
(2) The inlet pressure is about $3.62 \mathrm{E}+02 \mathrm{~Pa}$, the outlet1 is $2.54 \mathrm{E}+01 \mathrm{~Pa}$, outlet2 is $4.3 \mathrm{E}+01 \mathrm{~Pa}$, outlet 3 is $3.39 \mathrm{E}+01 \mathrm{~Pa}$, and outlet 4 is $3.24 \mathrm{E}+$ $01 \mathrm{~Pa}$;

(3) The condition has been improved on the gathering pressure the curvature is increased at the outlet of the manifold; the outside pressure is slightly larger than the inside, but it is almost the same.

The velocity field is analyzed of the manifold by using the velocity vector. The optimized velocity vector of the manifold is shown in Fig. 10.

What can be obtained is as follows:

(1) The velocity of flow is increased progressively from the inlet to the outlet;

(2) The gas velocity increases in each intake manifold due to the smaller resistance after optimization. The inlet speed is about $14 \mathrm{~m} / \mathrm{s}$, the manifold 1 outlet speed is $27.9 \mathrm{~m} / \mathrm{s}$, the manifold 2 is $25.8 \mathrm{~m} / \mathrm{s}$, the manifold 3 is $31.2 \mathrm{~m} / \mathrm{s}$, and the manifold 4 is $26.3 \mathrm{~m} /$;

(3) The flow velocity is larger in the outside than inside of the intake manifold; however, the degree is much better than the before.

The air flow is shown in Table 3 through simulating and calculation.

Known from the table:

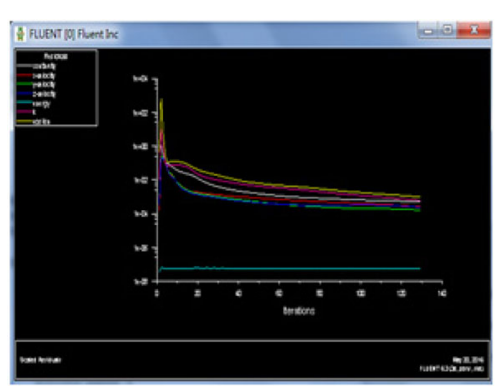

manifold 1 residual plot

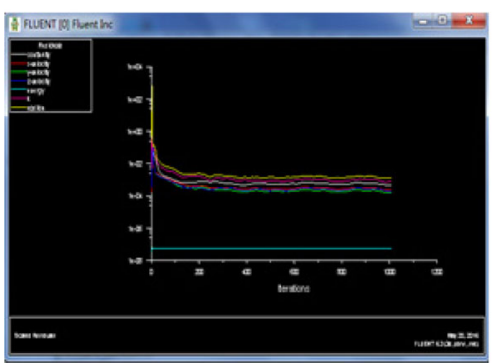

manifold 3 residual plot

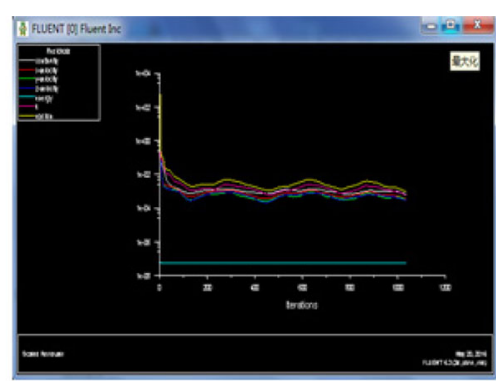

manifold 2 residual plot

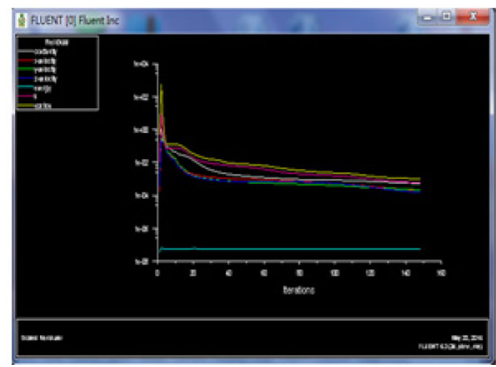

manifold 4 residual plot

Fig. 8 Improved intake manifold residual map 


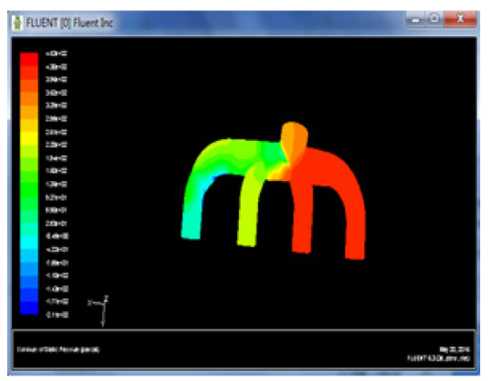

manifold 1 pressure cloud

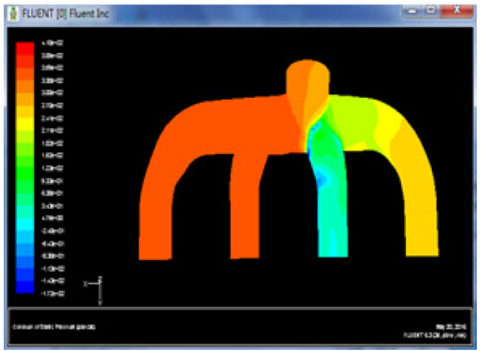

manifold3 pressure cloud

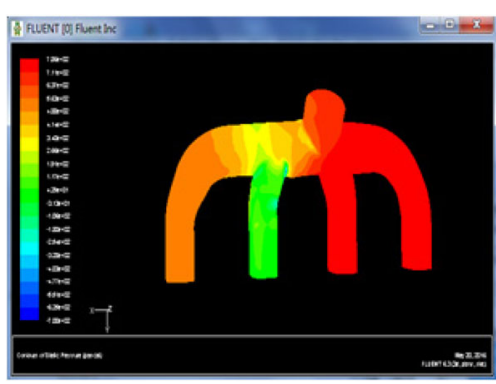

manifold 2 pressure cloud

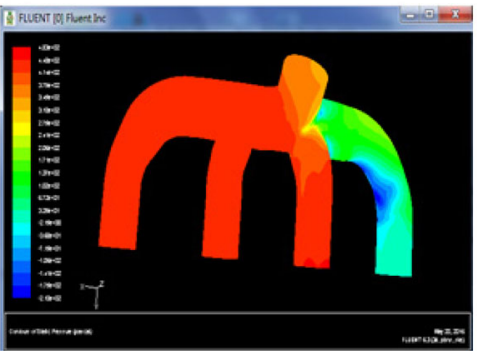

manifold 4 pressure cloud

Fig. 9 Intake manifold pressure cloud

(1) The air flow is increased obviously in the manifold after optimization;

(2) Non-uniformity is reduced to $7.48 \%$ about the inlet air. The other manifolds are more uniform except that the intake air flow is a slightly larger in the manifold 4. The cause of the vortex flow in the surge tank is the air flow that cannot enter the manifold during the air intake process, which hinders the flow more or less.

(3) The air intake volume is the least because the manifold 3 is closest to the intake port, and it has the greatest resistance; the air intake volume decreases from manifold 1 to manifold 3; and the

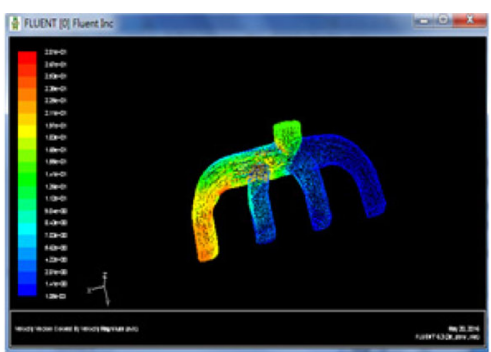

manifold 1 speed vector

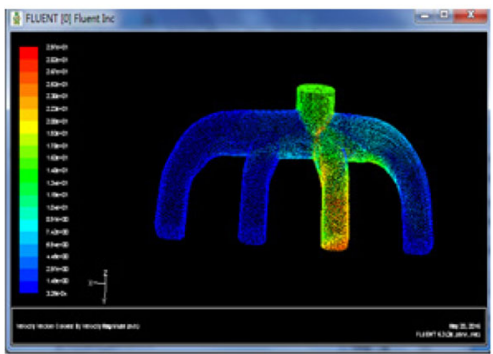

manifold 3 speed vector

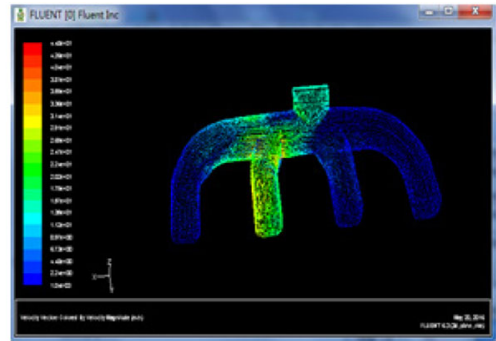

manifold 2 speed vector

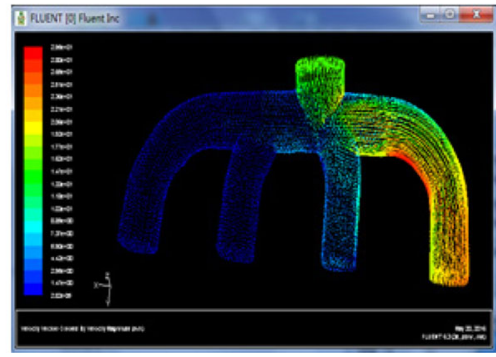

manifold 4 speed vector

Fig. 10 Intake manifold speed vector 
Table 3 Intake manifold air flow

\begin{tabular}{lllll}
\hline The number & 1 & 2 & 3 & 4 \\
\hline Flow $Q\left(10^{-2} \mathrm{~kg} / \mathrm{s}\right)$ & 3.61 & 3.52 & 3.43 & 3.78 \\
\hline
\end{tabular}

air intake is increased dramatically in the manifold 4 because it is the farthest from the air intake and closed to the tank wall, the vortex cannot be formed.

\section{Conclusion}

In this paper, the $3 \mathrm{D}$ model of the intake manifold is reconstructed using the inverse method and CATIA software. The applicability and authenticity of the model is ensured due to the actual shape of the engine intake manifold. The internal flow field of the manifold is analyzed by combining CAD, CATIA, and CFD software with FLUENT; in addition, the equation is established on the air flow in the manifold and used in the SIMPLE algorithm. The calculation speed is accelerated through combining GAMBIT and FLUENT software, dividing the internal flow field of the intake manifold, and setting the boundary conditions. The results show that the optimized flow field is reasonable, the air flow is higher, and the pressure is reduced to varying degrees in the manifold, which effectively reduces the intake resistance and helps to increase the intake manifold, especially in the main optimization position by comparing the internal flow field of the engine intake manifold and the optimized intake manifold.

\section{Abbreviations}

CAD: Computer-aided design; CFD: Computational fluid dynamics

\begin{abstract}
About the authors
Author1: Shuqing Guo female, associate professor, master tutor, has been working in Automotive Department at Transportation and Building Engineering College, Beihua University, China since 1999. My current research involves traffic environment, vehicle collision, and controlled technology. Presided over more than 10 projects at various levels, invention patent 2, and published more than 20 papers. Author2: Shuo Huang male, was born in 1992, pursuing master student, is learning in Automotive department at Transportation and Building Engineering College, Beihua University, research direction: Liquid control and the energy management strategy on new energy vehicle, participate in the project more than two, and published one paper.

Author 3: Mingshan Chi male, was born in 1997, pursuing master student, École Nationale Supérieure des Industries Chimiques. Research direction: Fluid mechanics.
\end{abstract}

Availability of data and materials

Please contact the authors for data requests.

\section{Authors' contributions}

Guo Shuqing-writing the whole paper and research on the simulation of the intake manifold. Huang Shuo-research on the reversing engineer and research on the method of the improved design of the engine intake manifold. Chi Mingshan-research on the aerodynamics and liquid mechanics and to create an analytical model, and checking the entire article. All authors read and approved the final manuscript.

\section{Competing interests}

The authors declare that they have no competing interests.

\section{Publisher's Note}

Springer Nature remains neutral with regard to jurisdictional claims in published maps and institutional affiliations.

\section{Author details}

${ }^{1}$ Faculty of Traffic and Building Engineering, Beihua University, Jilin City, China. ${ }^{2}$ École Nationale Supérieure des Industries Chimiques, Nancy, France.

Received: 29 April 2018 Accepted: 9 July 2018

Published online: 06 August 2018

References

1. Y Yongsheng, Z Liangliang, D Wu, Research on multipoint forming of automobile cover based on reverse engineering. Automobile Technology (06), 58-61 (2012)

2. Z Canming, Z Xuexun, J Meixia, W Wang, L Shuangli, application of reverse engineering in the design of automobile disc braking system [J]. Automobile Science \& Technology (01), 12-15 (2016)

3. G Wei, CFD analysis andexperimental study on intake system of the engine[D] (Harbin institute of Technology, 2012)

4. W Fujun, Computational Fluid Dynamics Analysis[M] (Tsinghua University Press, 2004)

5. W Ruijin, Z Kai, FLUENT Technology Foundation and Application Examples[M] (Tsinghua University Press, 2007)

6. A Lanzotti, F Renno, M Russo, R Russo, M Terzo, Design and development of an automotive magnetorheological semi-active differential[J]. Mechatronics 24(5), 426-435 (2014)

7. D Hao, Research on CFD-assisted engine intake pipe[J]. Equipment Manufacturing Technol (02), 17-20 (2010)

8. L Ming, Numerical Simulation of the Effect of Variable Intake on Gasoline Engine Flow Characteristics and Performance[D] (Tianjin University, 2006)

9. X He, Z Tingfang, Z Chaomin, S Wei, Three-dimensional design of automobile body based on reverse engineering[J]. J Nanchang Univ (Engineering \& Technol Ed) 37(02), 128-131+141 (2015)

10. WK Ham, M Ko, SC Park, a framework for simulation-based engine-control unit inspection in manufacturing phase[J]. Control. Eng. Pract. 59, 137-148 (2017)

11. T Guofu, Z Zhisheng, Reverse Design of Vehicle Body Backplate Based on CATIA[J]. Automobile Engineer (04), 39-42 (2012)

12. Liu Jifeng, Fan Xiangmei, Liu Duanxiao, "Application of reverse design based on CATIA in design of automotive interior parts". Auto Engineer. (03):52-54 (2011)

13. K Abdil, Implementation of 3D optical scanning Technology for Automotive Applications[J]. Sensors 9(3), 1967-1979 (2009)

14. SR Sukumar, P Govindasamy, AF Koschan, DL Page, MA Abidi, Imaging-based thermal modelling and reverse engineering of as-built automotive components: a case study[J]. Virtual and Physical Prototyping 5(1), 21-32 (2010)

15. Z Yu, The car body reverse design method based on CATIA. J Anshan Normal Univ (04), 15-18 (2011)

16. C Xiaoxing, On the Key Technology and Application to Reconstruct Reverse Engineering Model[D] (Zheng Zhou University, 2012)

17. H Zehao, Numerical analysis on the engine intake manifold flow field[J]. Machinery Design and Manufacture (02), 156-158 (2012)

18. F Aihua, Flow field analysis and structure improvement based on CAD/CFD plastic intake manifold[D] (Harbin institute of Technology, 2011)

19. M Vanco, G Brunnett, Direct segmentation of algebraic models for reverse engineering[J]. Computing, 207-220 (2004)

20. Y Chengliang, Variable intake manifold surface reconstruction and fluid analysis based on point-cloud [D] (Harbin institute of Technology, 2006) 